\title{
2 core experiential and 4 behavioural dimensions of the initial prodrome in schizophrenia emerged
}

Møller P, Husby R. The initial prodrome in schizophrenia: searching for naturalistic core dimensions of experience and behavior. Schizophr Bull 2000;26:217-32.

QUESTION: What are the core experiential and behavioural dimensions of the initial prodrome in schizophrenia?

\section{Design}

Qualitative design using open ended, indepth interviews.

\section{Setting}

A specialised schizophrenia unit in Oslo, Norway.

\section{Participants}

19 Norwegian speaking patients (mean age 22 y, 58\% men) with a first episode of DSM-III-R and DSM-IV schizophrenia or schizophreniform disorder. Exclusion criteria were medical or neurological illness; brain lesion; mental retardation; or $>2$ years since initiation of first adequate treatment.

\section{Methods}

Interviews were done as soon as diagnosis was established. Patients and families reported separately all possible prodromal experiences and behaviours. During the 5-8 hour interviews, repeated question and answer cycles were used to increasingly clarify phenomena. Transcripts, records, reports, diaries, and notes were condensed according to a conceptual framework. Data saturation was reached when 12-14 interviews were finished.

\section{Main findings}

The estimated mean prodrome duration was 127 weeks, and the median estimate was 50 weeks. Distinct qualitative changes of experience were reported long before behavioural consequences created serious family concern. Most patients reported having had serious difficulty interpreting and communicating their early experiential changes.

8 experiential dimensions of the prodrome emerged, among which 2 were highlighted as tentative core dimensions: (1) disturbance of perception of self (eg, painful emotional indifference and distance to self, feeling of unreality or strangeness), and (2) extreme preoccupation by and withdrawal to overvalued ideas (eg, exaggerated brooding and introspection, extremely occupied by thought about how to be good enough).

4 potential dimensions of prodromal behaviour were: (1) quitting school, university, or job, or major school truancy, (2) marked and lasting observable shift of interest (ie, from practical, social, organisational interests to contemplative, introverted, and individual interests), (3) marked and lasting social passivity, withdrawal, or isolation, and (4) marked and lasting change in global appearance or behaviour.

\section{Conclusion}

8 experiential and 4 behavioural dimensions of the initial prodrome in schizophrenia emerged, among which 2 were suggested as core experiential dimensions.

\section{COMMENTARY}

In this study, which gathers patients' memories of the prodromal phase of their schizophrenia, Møller and Husby make an important contribution to our knowledge base and potentially, our clinical practice. Naturalistic, anthropological research on severe mental illness is notoriously difficult to do and analyse. As with other intensive small group studies, complexity and fine grained detail counterbalance the loss of statistical rigour and reproducibility.

By using exhaustive, multisource interviews of participants' and families' recollections of the earliest days of symptoms and behaviour changes, the authors provide us with a level of detail rarely found. Using a group of patients whose prodromal period is relatively recent, they are able to identify core dimensions shared among patients of different ages, backgrounds, and premorbid histories.

Naturally, one of the greatest challenges facing us as clinicians and public health agents is the timely and accurate identification of schizophrenia in its earliest manifestations. The existing literature tends to provide only highly general descriptions and clinical, rather than first person, accounts of illness onset. These authors begin the process of categorising and more rigorously specifying the essential characteristics of prodromes by allowing us to share in the subjective accounts of their research participants.

This study documents an extremely long period in which participants report alterations in their subjective experience of themselves before the development of noticeable behavioural changes. The 2 areas of such alterations reported (ie, shifts in their perception and feelings about themselves, and extreme preoccupation by and withdrawal to overvalued ideas) were present in all of the 19 Norwegian schizophrenic patients studied, underscoring the need to take such reports quite seriously, even in otherwise well functioning patients. We currently have no documented means of arresting, or even attenuating, the development of schizophrenia. By beginning family and patient education earlier and increasing our vigilance for signs of behavioural changes, we can almost certainly help to reduce the pain, suffering, and social price of undiagnosed illness.

Stephen M Goldfinger, MD State University of New York/Health Science Center, Brooklyn Brooklyn, New York, USA 